\title{
Assessment of CB agonist CP55940 in maturity for rat hippocampal neurons using a high-throughput immunocytochemical assay and image digital analysis
}

\author{
Mitsuoka Toshinari $^{1}$, Shogo Mase ${ }^{1,2}$, Noriko Koganezawa ${ }^{2}$, Yuichi Katou ${ }^{1}$, Izuo Tutui ${ }^{1}$, \\ Tomoaki Shirao $^{2}$, Yuko Sekino ${ }^{1}$
}

${ }^{1}$ Lab. HCBDD, Grad. Sch. Pharm, Univ Tokyo, ${ }^{2}$ Dept Pharmacol, Gunma Univ, Grad Sch Med.

Cannabinoids are psychoactive ingredients and naturally occurring compounds found in the Cannabis sativa plant. The cannabis disrupts normal brain function in the long term, especially for young people who are still in the developmental stage. Cannabinoids exert their effects by interacting with cannabinoid receptors (CB1and CB2) present on the surface of cells in different parts of the central nervous system. Cannabinoid receptor agonists act presynaptically to inhibit the release of glutamate. We have shown that NMDA receptor activation reduced the amount of an actin-binding protein drebrin in postsynaptic sites. In this study, we assessed effects of CP55940 (CB1 and CB2 agonist) at the developmental stage using a high-throughput immunocytochemical assay and image digital analysis. Hippocampal neurons prepared from embryonic rats (SKY neuron,Altmed,Inc.Tokyo) were incubated in 96well microplates. After 7, 14 or 21days, cultured neurons were administrated with CP55940. Then treated with 10 and $100 \mu \mathrm{M}$ glutamate for $10 \mathrm{~min}$ in 21 days. After fixation, cultured neurons were immunostained with anti-drebrin and anti-MAP2 antibodies. The numbers of cells and drebrin clusters, the length of dendrites, and the fluorescence intensity of drebrin clusters were automatically quantified with image digital processing using a protocol that we originally developed. CP55940 significantly increased the numbers of drebrin clusters and the length of dendrites. These results suggested that CP55940 may affect the function of neurons. 